\title{
A Prospective Study to Assess the Optimal Incubation Times for Culture and Aerobic Bacterial Profile in Prosthetic Joint Infections
}

\author{
Varsha Gupta ${ }^{1}$ Mandeep Kaur ${ }^{2, \bullet}$ Prapti Bora ${ }^{1}$ Pooja Kumari ${ }^{1}$ Priya Datta ${ }^{3}$ Ravi Gupta ${ }^{4}$ \\ Jagdish Chander ${ }^{1}$ \\ ${ }^{1}$ Department of Microbiology, Government Medical College \\ Hospital, Chandigarh, Punjab, India \\ ${ }^{2}$ Department of Microbiology, Adesh Institute of Medical Sciences \\ and Research, Bathinda, Punjab, India \\ ${ }^{3}$ Department of Medical Parasitology, Postgraduate Institute of \\ Medical Education and Research, Chandigarh, Punjab, India \\ ${ }^{4}$ Department of Orthopedics, Government Medical College \\ Hospital, Chandigarh, Punjab, India

\begin{abstract}
Address for correspondence Mandeep Kaur, MBBS, MD, Department of Microbiology, Adesh Institute of Medical Sciences and Research, Bathinda, Punjab 151001, India (e-mail: drmandeepkaur@gmail.com).
\end{abstract}

J Lab Physicians 2021;13:224-230.

\begin{abstract}
Keywords

- prosthesis

- infections

- culture

- prolonged incubation

- Staphylococcus aureus
\end{abstract}

Introduction With an increase in the number of total joint arthroplasties, the count of prosthetic joint infections (PJIs) is also increasing and has become a nightmare for an orthopaedic surgeon. Microbiological diagnosis is important for administering definitive antimicrobial treatment. Negative culture reports hamper patient management and prolonged incubation periods have increased the culture yield but at the risk of culture contamination in conventional microbiology settings. Thus, we aimed to optimize the best incubation time for culture and the aerobic bacterial profile of PJIs. Material and Methods Over a year, samples from clinically suspected PJI patients were collected and processed for culture using standard techniques. The samples were incubated for up to 10 days with daily subculturing on to solid media. The bacterial isolates were identified and antibiotic susceptibility was performed.

Results Out of 200 patients, 105 were included in the study and samples were collected in triplicate. In 70 cases culture was positive and 35 were culture negative PJIs. Mean incubation days to culture positivity was 3.6 days with $97.14 \%$ culture positivity seen by the seventh day of incubation. Twenty-four percent methicillin-resistant Staphylococcus aureus, and more than $50 \%$ extended spectrum $\beta$ lactamase producing Klebsiella pneumoniae and Escherichia coli were isolated.

Discussion Culture positivity in PJIs provides definitive evidence of infection and guides the treatment. Increasing the incubation times can help in maximizing the culture yield, and we found that $97.14 \%$ pathogens grew within 7 days of incubation. Prolonging it further would not provide an added advantage especially in a resource-constraint setting. published online July 6, 2021
DOI https://doi.org/

$10.1055 / \mathrm{s}-0041-1730879$

ISSN $0974-2727$ (c) 2021. The Indian Association of Laboratory Physicians.

This is an open access article published by Thieme under the terms of the Creative Commons Attribution-NonDerivative-NonCommercial-License, permitting copying and reproduction so long as the original work is given appropriate credit. Contents may not be used for commercial purposes, or adapted, remixed, transformed or built upon. (https://creativecommons.org/licenses/by-nc-nd/4.0/).

Thieme Medical and Scientific Publishers Pvt. Ltd. A-12, 2nd Floor, Sector 2, Noida-201301 UP, India 


\section{Introduction}

The demand for total joint arthroplasty (TJA) is significantly rising in India because of increased elderly population, trained orthopaedic surgeons, sedentary lifestyle, booming economy, improved hospital infrastructures, and emerging medical tourism. Moreover, the recent price capping of the medical devices by the central government has made total knee arthroplasty (TKA) affordable for the common man. ${ }^{1}$ Even with the best precautions, prosthetic joint infections (PJIs) do occur and have been posing a significant burden on orthopaedic services due to the sheer number of patients with hip and knee prostheses. The incidence of PJI after primary interventions in total hip arthroplasty (THA) or TKA is 1.5 to $2.5 \%$ but may increase to higher rates (2-20\%) when revision procedures are performed. A single institutional study on PJIs from northern India have reported a cumulative incidence of $1.1 \%{ }^{1,2}$

The clinical spectrum of PJIs is variable and clinical signs and symptoms include pain, joint swelling or effusion, erythema, fever, drainage, or a discharging sinus. ${ }^{1}$ The Musculoskeletal Infection Society (MSIS) proposed a definition for PJI in 2015 which later was revised in 2018; it can be universally adopted for PJI diagnosis., ${ }^{3,4}$ As per the 2015 definition, definite PJI diagnosis could be made if one of two major pathognomonic criteria or four of six minor criteria are met while later in 2018 it was revised to scoring-based system where operative criteria were used to fulfill the criteria for PJI in case of inconclusive scores in minor criteria (-Table 1). It is important to identify the infecting organism responsible for successful treatment outcomes in PJIs by administration of appropriate and specific antimicrobial agents. Microbiological culture remains the standard technique for the identification of the infecting microorganism, but the frequency of negative culture results ranges from 7 to $23 \%{ }^{3}$ The biofilm formation especially in low-grade arthroplasty infection on the implant by sessile bacteria is one of the major pathogenic factors responsible for PJIs as well as for culture negativity where bacteria enmeshed within the biofilm fail to grow on culture. The biofilms further increase the problem of antibiotic drug resistance, hence making it more important to isolate the infecting pathogen and determine its antimicrobial susceptibility for optimal patient management. Routinely in most of the laboratories in India, the culture reports are dispatched in 48 to 72 hours and the chances are high that most of the prosthesis-related infections remain undiagnosed microbiologically. In particular, it seems that the need for an appropriate period of incubation has been underestimated. Many studies have suggested prolonging the culture incubation periods up to 14 days in joint infections. ${ }^{5,6}$ But keeping culture for prolonged times in a conventional laboratory set up will further increase the isolation of contaminants rather than the pathogens. Hence this study was planned to assess the optimal incubation times for cultures in PJIs in conventional culture settings and to study the aerobic bacterial profile of PJIs so as to design optimal antibiotic therapy for patients.

Table 1 MSIS criteria for PJI diagnosis 3,4

\begin{tabular}{|c|c|c|c|c|}
\hline & 2015 MSIS criteria & \multicolumn{3}{|c|}{2018 MSIS criteria } \\
\hline Major criteria & $\begin{array}{l}\text { 1. A sinus tract communicating with the } \\
\text { prosthesis. } \\
\text { 2. A pathogen is isolated by culture from two } \\
\text { separate tissue or fluid samples obtained } \\
\text { from the affected prosthetic joint. }\end{array}$ & \multicolumn{3}{|c|}{$\begin{array}{l}\text { 1. A sinus tract communicating with the prosthesis. } \\
\text { 2. A pathogen is isolated by culture from two separate tissue or fluid } \\
\text { samples obtained from the affected prosthetic joint. }\end{array}$} \\
\hline Interpretation & Any one of the above is definite PJI. & \multicolumn{3}{|c|}{ Any one of above is definite PJI. } \\
\hline \multirow[t]{6}{*}{ Minor criteria } & \multirow{6}{*}{$\begin{array}{l}\text { 1. Elevated serum ESR and CRP concentration. } \\
\text { 2. Elevated synovial leukocyte count. } \\
\text { 3. Elevated synovial PMN\%. } \\
\text { 4. Presence of purulence in the affected joint. } \\
\text { 5. Isolation of a microorganism in one culture } \\
\text { of periprosthetic tissue or fluid. } \\
\text { 6. Greater than five neutrophils per high-power } \\
\text { field in five high-power fields observed from } \\
\text { histologic analysis of periprosthetic tissue at } \\
\times 400 \text { magnification. }\end{array}$} & \multicolumn{2}{|r|}{ Preoperative diagnosis } & Score \\
\hline & & Serum & $\begin{array}{l}\text { Elevated CRP or D-dimer } \\
\text { Elevated ESR }\end{array}$ & $\begin{array}{l}2 \\
1\end{array}$ \\
\hline & & Synovial & $\begin{array}{l}\text { Elevated synovial WBC count or LE } \\
\text { Positive } \alpha \text {-defensin } \\
\text { Elevated synovial PMN (\%) } \\
\text { Elevated synovial CRP }\end{array}$ & $\begin{array}{l}3 \\
3 \\
2 \\
1\end{array}$ \\
\hline & & Score & \multicolumn{2}{|l|}{$\begin{array}{l}\geq 6 \text {-infected } \\
2-5-\text { possibly infected } \\
0-1-\text { not infected }\end{array}$} \\
\hline & & \multicolumn{2}{|c|}{ Intraoperative diagnosis ${ }^{\mathrm{a}}$} & \\
\hline & & \multicolumn{2}{|c|}{$\begin{array}{l}\text { Preoperative score } \\
\text { Positive histology } \\
\text { Positive purulence } \\
\text { Single positive culture }\end{array}$} & $\begin{array}{l}- \\
3 \\
3 \\
2\end{array}$ \\
\hline Interpretation & Four out of six criteria met is definite PJI. & Score & \multicolumn{2}{|l|}{$\begin{array}{l}\geq 6 \text {-infected } \\
4-5-\text { inconclusive } \\
\leq 3-\text { not infected }\end{array}$} \\
\hline
\end{tabular}

Abbreviations: CRP, C-reactive protein; ESR, erythrocyte sedimentation rate; LE, leucocyte esterase; PJI, prosthetic joint infection; PMN, polymorphonuclear; WBC, white blood cell.

aFor inconclusive minor criteria patients, operative scores may fulfill definition of PJI.

${ }^{\mathrm{b} C o n s i d e r}$ for molecular diagnosis in these cases like next generation sequencing. 


\section{Materials and Methods}

This prospective observational study was conducted for a period of one year in the Departments of Microbiology and Orthopaedics at Government Medical College Hospital, Chandigarh after due approval from Institutional Research and Ethics Committee of our hospital. The clinically suspected PJI patients meeting 2015 MSIS diagnostic criteria (minimum one major criteria or four minor criteria) were included in the study after taking due informed consent. From these patients, at least three samples that include joint fluid or aspirate, bone fragments, pus, synovial tissue samples were aseptically collected, transferred into the aerobic blood culture bottles (Hi-media, Mumbai, India), and sent for culture and sensitivity. The samples were not tested for the presence of anaerobes. The bone fragments and synovial tissue were cut into small bits/crushed with the sterile pestle and mortar before inoculation into the culture bottles. All clinical and laboratory procedures were performed as per standard guidelines. ${ }^{7,8}$ In the laboratory, the culture bottles were incubated under aerobic conditions at $37^{\circ} \mathrm{C}$ and daily subcultures were made on $5 \%$ sheep blood agar and MacConkey for 7 days until the microbial growth was obtained and the last subculture being performed at tenth day of incubation before reporting the culture results as sterile. The maximum incubation period of 10 days was taken based on our observation in a pilot study conducted on 20 suspected PJI samples in which out of 20 samples, 12 grew pathogenic microorganism within 10 days while eight remained sterile even after 15 days of incubation. The cultural isolates obtained were identified using standard microbiological techniques. ${ }^{9}$ The culture showing growth of similar pathogen with similar antimicrobial profile isolated from at least two samples was taken as a definitive pathogen causing infection. For all culture positive cases, the antimicrobial susceptibility testing of the bacterial isolates obtained was performed and interpreted as per CLSI Guidelines, 2018. The antimicrobials tested against gram-positive isolates were penicillin (10IU), cefoxitin
(30 $\mu \mathrm{g})$, erythromycin $(15 \mu \mathrm{g})$, clindamycin $(2 \mu \mathrm{g})$, cotrimoxazole $(1.25 / 23.7 \mu \mathrm{g})$, linezolid $(30 \mu \mathrm{g})$, ciprofloxacin $(5 \mu \mathrm{g})$, teicoplanin $(30 \mu \mathrm{g})$, gentamicin $(10 \mu \mathrm{g})$, doxycycline $(30 \mu \mathrm{g})$ and for gram-negative bacteria, cefepime (30 $\mu$ g), amoxycillin clavulanic acid $(30 / 20 \mu \mathrm{g})$, ciprofloxacin $(5 \mu \mathrm{g})$, piperacillin tazobactam $(100 / 10 \mu \mathrm{g})$, amikacin $(30 \mu \mathrm{g})$, ceftazidime (30 $\mu \mathrm{g})$, ceftriaxone $(30 \mu \mathrm{g})$, imipenem $(10 \mu \mathrm{g})$, tetracycline $(30 \mu \mathrm{g})$, cefotaxime $(30 \mu \mathrm{g})$, tobramycin $(10 \mu \mathrm{g})$, ampicillin sulbactam $(10 / 10 \mu \mathrm{g})$ were tested (HiMedia, Mumbai, India). The multidrug resistant (MDR) isolates were phenotypically characterized as per their antibiograms into methicillin-resistant Staphylococcus aureus (MRSA), vancomycin-resistant enterococci, metallo $\beta$-lactamase (MBL), extended spectrum $\beta$ lactamase (ESBL) producers. As per the CLSI guidelines, the methicillin resistance in Staphylococcus was determined by cefoxitin disk $(30 \mu \mathrm{g})$ method while vancomycin resistance was tested by vancomycin screen agar. MBL production in gram-negative bacteria was tested by disk synergy method and ESBL production was confirmed by disk potentiation test using ceftazidime $(30 \mu \mathrm{g})$, and cefotaxime (30 $\mu \mathrm{g})$ antibiotic disks with or without clavulanic acid $(10 \mu \mathrm{g}) .{ }^{10}$

\section{Results}

\section{Infection and Bacterial Isolates}

Out of 200 clinically suspected PJI patients, 105 patients met the MSIS criteria and were included in the study. From these patients, samples that included 51 wound discharge or pus, 33 joint fluid aspirates, 10 bone fragments, and 28 synovial tissue samples were collected in triplicate and were processed by aerobic culture methods. Seventy-five patients were male and the rest female. The age group of PJI patients ranged from 12 to 76 years with mean age being 44.5 years. Definitive evidence of PJI by culture positivity was observed from 70 patients (66.67\%) in which at least two out of three samples grew same bacterial pathogen on culture while in 35 samples, no pathogen grew on culture even after 10 days of incubation.

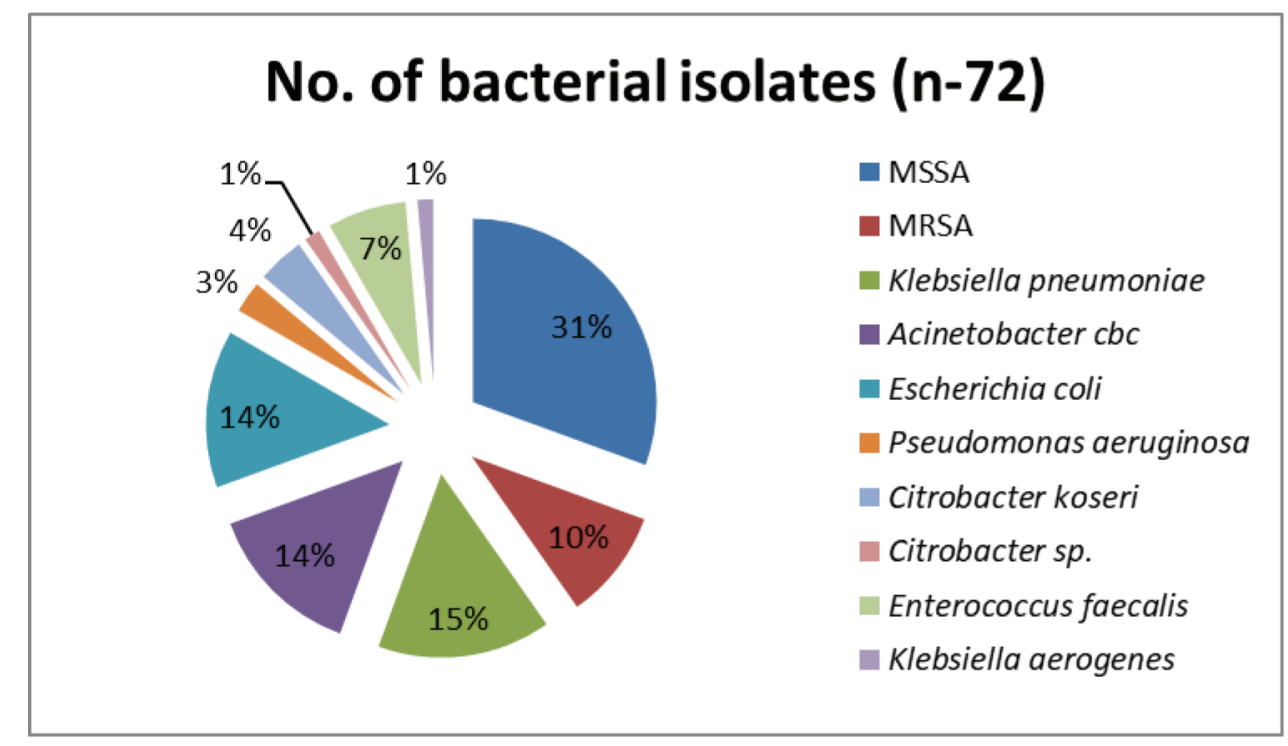

Fig. 1 The profile of bacterial pathogens in prosthetic joint infections. 
The most commonly involved joint in device-related infection was knee joint (54\%; 57/105) followed by hip joint (40\%; 42/105) while shoulder and ankle joint were involved in rest $(5.7 \% ; 6 / 105)$ of the cases. Highest isolation rate of pathogenic bacteria was from pus samples (82.35\%; 42/51) followed by synovial tissue (53.58\%; $15 / 28)$, bone (40\%; 4/10), and joint fluid or aspirate (27.27\%; 9/33) (-Fig. 1). Seventy-two bacterial strains were isolated from culture positive cases among which Staphylococcus aureus was the most frequently isolated pathogen $(40.28 \% ; n-29)$, followed by Klebsiella pneumoniae (15.27\%; $n-11)$, Acinetobacter CBC (13.89\%; $n-10)$, and E. coli (13.89\%; $n-10)$ (-Fig. 2).

\section{Time of Diagnosis of Infection by Culture}

The mean number of days until bacterial growth became detectable in infected samples was 3.6 days in our study and it did not differ much among different clinical samples except in bone fragments, the mean and median incubation period for culture positivity was observed as 5 days. Overall, maximum culture positivity was seen on 3rd day of incubation (32.85\%; $n-23 / 70)$ while it was $25.71 \%$ (18/70) on day 4 and $14.28 \%(10 / 70)$ on day 5. Cumulatively, 92.85\% (65/70) of culture grew pathogens within 5 days of incubation period and $97.14 \%$ (68/70) by day 7 (-Fig. 3). Gram staining could reveal significant findings in only 25 cases out of 70 culture positivity with overall sensitivity of only $35.71 \%$.

\section{Antimicrobial Drug Susceptibility}

S aureus was the most common organism isolated in our study and showed only $4 \%$ susceptibility to penicillin, ciprofloxacin (44\%), clindamycin (44\%), erythromycin (48\%), linezolid (99.07\%), gentamicin (78\%), and 100\% susceptibility toward vancomycin and teicoplanin. MRSA constituted $24.13 \%(n-7)$ of total $29 \mathrm{~S}$. aureus isolates and vancomycin resistance was not observed among gram-positive cocci. Acinetobacter CBC showed only $22 \%$ susceptibility toward ceftazidime while imipenem was found to be susceptible in 56\% cases. $78 \%$ susceptibility was observed for tobramycin, amikacin, and ampicillin sulbactam and ciprofloxacin was susceptible in $44 \%$ cases. The percentage susceptibility observed among other gram-negative bacilli isolated was $40 \%$ for tetracycline, piperacillin-tazobactam (20\%), imipenem (70\%), amikacin (30\%), ceftazidime (05\%), ceftriaxone (15\%), cefepime (10\%), amoxiclav (15\%), and ciprofloxacin (30\%). ESBL production was seen in $54 \%(6 / 11)$ isolates of $K$. pneumoniae and $50 \%$ (5/10) E. coli, while $36.36 \%$ (4/11) of K. pneumoniae, 30\% E. coli (3/10) and 30\% Acinetobacter CBC (3/10) were MBL producers. Amp $C$ production was observed in one isolate each of K. pneumoniae, E. coli, and Acinetobacter CBC. Among K. pneumoniae and E. coli isolates, co-expression of the three enzymes (ESBL, MBL, and Amp C) was seen in one isolate each.

\section{Discussion}

PJIs are uncommon and occur in only 1 to $2 \%$ of patients with hip and knee replacements and in up to $6 \%$ of patients with internal fixation of closed fractures. ${ }^{6}$ Acquisition of PJI can occur by different mechanisms. Most common is the direct seeding of microorganisms during surgery whereby after the initial contact (either direct or aerosolized contamination of the prosthesis or periprosthetic tissue [PPT]), microorganisms colonize the surface of the implant. As per many studies, the bacterial concentration needed to induce an infection is significantly reduced in the presence of the prosthetic material. Moreover, the presence of prosthesis can lessen the neutrophil activity thereby increasing the infection susceptibility. Other mechanism of infection initiation is through contiguous spread of infection from an adjacent site or by hematogenous seeding. ${ }^{1}$

The management of PJI cases is difficult and a reliable microbiological diagnosis is crucial for determining appropriate treatment. It is a gold standard test for PJI diagnosis,

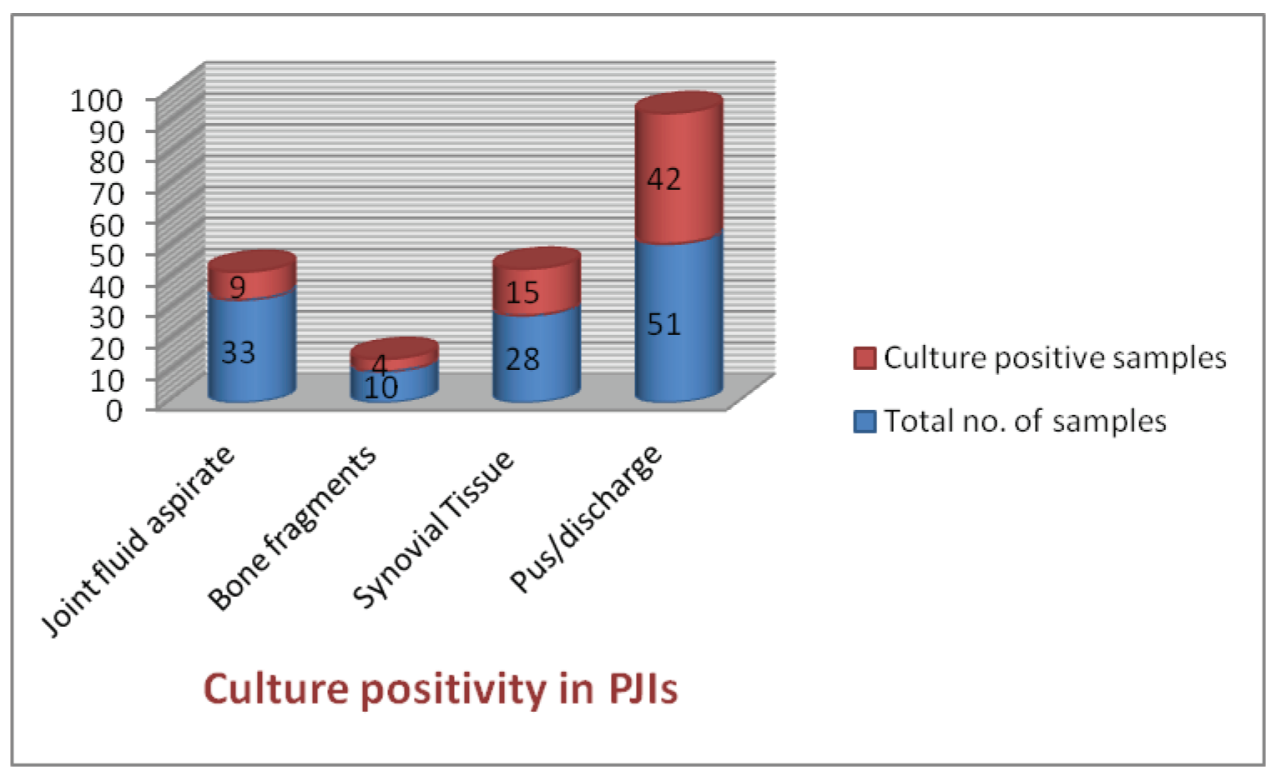

Fig. 2 Sample wise culture positivity in prosthetic joint infections. 


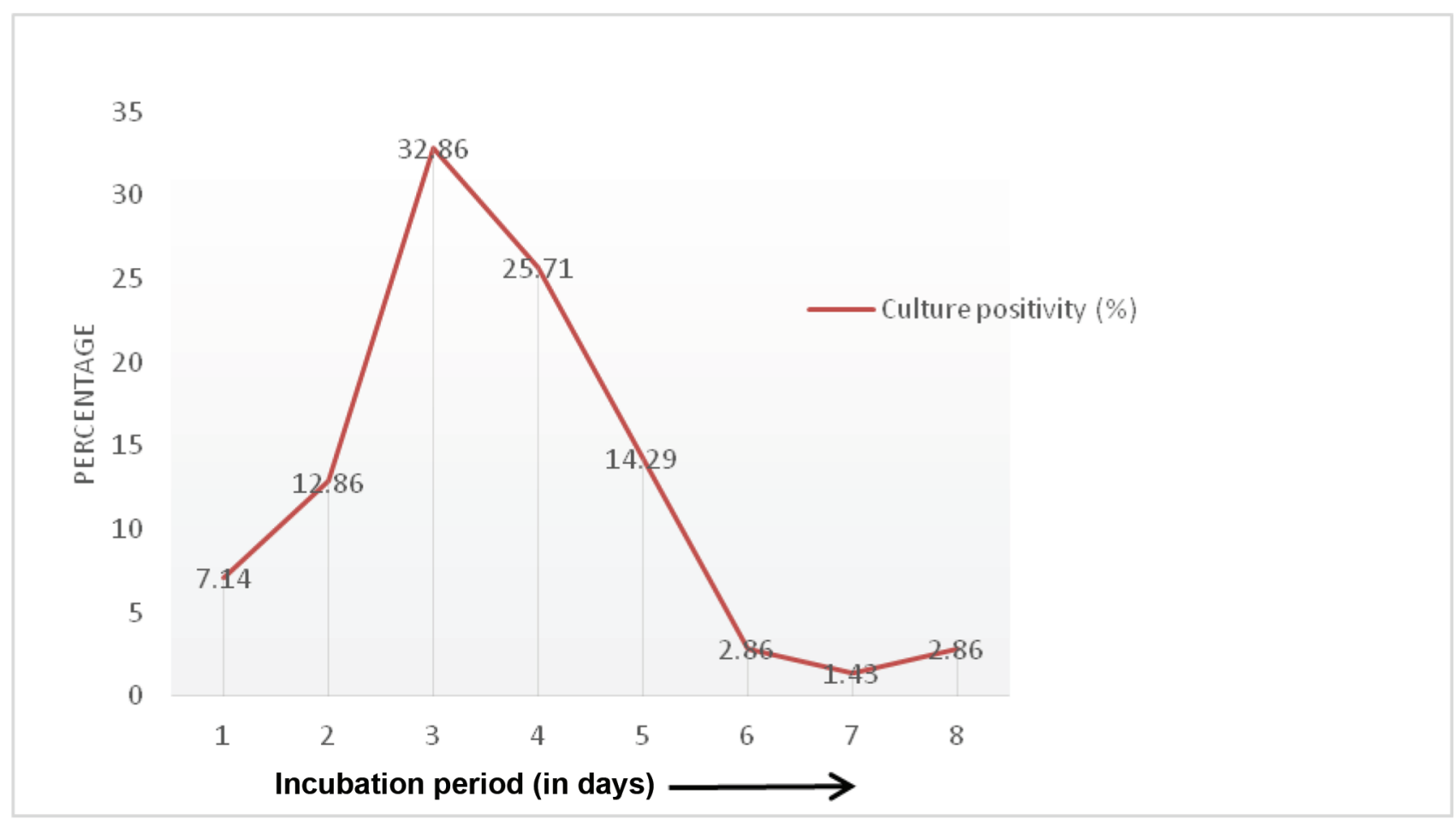

Fig. 3 Time to culture positivity in prosthetic joint infections.

but conventional PTT cultures have low sensitivity due to many factors like prior antibiotic exposure, the presence of viable but uncultivable organisms, slow growing organisms, inadequate incubation periods for culture, and the presence of biofilms rendering cultures sterile. The culture-negative PJI is a very perplexing condition to manage for the surgeon, patient, and infectious disease team. ${ }^{6,11}$ In recent years, the prevalence of culture-negative PJI has been on the rise and the traditional modalities for isolation of an infecting organism have failed in as many as $45 \%$ of patients. ${ }^{12}$ Withholding the antibiotic administration until specimens for culture has been obtained may help in culture isolation of the infecting organism, and if the arthroplasty is resected, the sonication fluid obtained after vortexing and sonication of the implant components may be an appropriate sample to be sent for culture. $^{8,13}$

Infectious Diseases Society of America (IDSA) recommends the collection of a minimum of three and optimally five or six PPT samples for both aerobic and anaerobic cultures at the time of revision surgery and each specimen should be obtained with a new sterile instrument. ${ }^{7}$ Despite withholding the preoperative antibiotics in cases of revision arthroplasty, the sensitivity of PPT culture has been determined to be $63 \%$ and the optimal incubation period for prosthetic joint samples remains controversial. One study has shown a 13-day incubation period to be optimal for recovery of slow growing organisms like Cutibacterium (Propionibacterium) species, while no supporting evidence was gathered from other studies. ${ }^{14}$

Very few studies have focused on finding out the optimal incubation time for orthopaedic surgical specimens and there are none so far from India. Most commonly, 5 to
7 days of incubation time in culture is used for PJI diagnosis with a recent proposal of prolonging it up to 14 days. Schäfer et al found a positive association of prolonged incubation period with increased proportion of positive samples and diversity of bacterial isolates, especially recovery of aerobic gram-positive rods, Propionibacterium spp., and Peptostreptococcus spp. were increased with 14-day incubation protocol. ${ }^{5}$ Similar findings were observed by Gunthard et al and Butler-Wu et al where prolonging the incubation period for more than 7 days resulted in increased culture positivity. ${ }^{15,16}$ Keeping the culture bottles for prolonged period is a difficult thing to follow in settings where conventional culture methods are being followed and with high throughput of clinical samples. Also, the risk of growing contaminants rather pathogens increase during frequent subculturing in conventional practices. Automated blood culture systems offer a great help in such a scenario. The present study showed that $97.14 \%$ culture positivity was observed within 7 days of incubation with a mean incubation period of 3.6 days. Incubation periods did not differ much among different types of samples except the mean and median incubation period was 5 days in bone fragment culture.

Gram stain is one of the most useful techniques for rapid diagnosis in the clinical microbiology laboratory and allows detecting bacteria and performing an initial classification of microbes based on their shape and staining characteristics. Its use in synovial fluid has been claimed to be highly specific, although sensitivity is only 40 to $45 \% .{ }^{17}$ In our study too, Gram staining did not offer great sensitivity and correlated well with culture in only $35.71 \%$. Apart from the diagnostic challenges, the management of these patients is a far more challenging issue, with scanty literature on the subject. ${ }^{18}$ 
The goals of PJI treatment involve the eradication of infection and restoration of the pain-free function of the infected joint. However, it may not be possible to achieve all these goals in every patient. For optimal treatment of PJI, both surgical intervention and antimicrobial therapy are needed and antimicrobial treatment without surgical intervention has not been routinely recommended. However, antibiotic suppression alone is reserved for patients with multiple comorbidities and not eligible for any surgical interventions, or for those patients who are unwilling for any surgery, and causative organisms are susceptible to oral antibiotics. Moreover, the antimicrobial therapy for PJI should be specific and based on the causative microorganism and its antimicrobial susceptibility pattern. ${ }^{1}$

The most common etiological agents involved in PJIs are S. aureus and S. epidermidis that account for close to $65 \%$ of PJIs. They are the most commonly reported microorganisms both in early and late infections as well as in TKA and THA. ${ }^{19}$ Salgado et al have reported S. aureus as causative organism in 33\% PJIs, out of which $24 \%$ were methicillin-sensitive S. aureus (MSSA) and $9 \%$ were MRSA. ${ }^{20}$ We observed similar results in our study with S. aureus implicated in $40.28 \%$ of PJI cases among whom 9.7\% were MRSA and 30.5\% were MSSA infections. In a review by Peel et al, MRSA contributed a much higher proportion (45\%) as the causative agent of PJIs. ${ }^{21}$ As per the global data, gram-negative bacteria are less commonly associated with PJI and account for only 6 to $23 \%$ of all episodes but the Indian studies on the other hand report high frequency of gram-negative PJIs that mostly include members of family Enterobacteriaceae and Pseudomonas aeruginosa. ${ }^{1,13}$ In our study, Klebsiella pneumoniae, E. coli, Klebsiella aerogenes contributed to $30 \%$ of PJIs followed by $13 \%$ by Acinetobacter CBC and P. aeruginosa in $2.78 \%$ infections.

The antimicrobial dug resistance is a common phenomenon observed in biofilm formation as the enmeshed bacteria in the extracellular matrix remain protected from antibiotics and evade the host defense mechanisms. So is the case in PJI where the implant frequently gets colonized with adherent microorganisms forming biofilms. In a study by Sebastian et al, $64 \%$ of the gram-negative isolates were multidrug resistant and methicillin resistance being observed among $34 \%$ of gram-positive isolates. ${ }^{1,13}$ In our study, $24 \%$ (7/29) of S. aureus isolates were MRSA and MDR gram-negative pathogens were isolated from PJIs that include 29.94\% ESBL (11/38), 26.31\% MBL (10/38), and 7.89\% (3/38) Amp C producers. A detailed list of suggested antimicrobial agents and their dosing for specific pathogens is available in IDSA guidelines for the management of PJIs. ${ }^{7}$ At our institute, vancomycin and teicoplanin showed $100 \%$ susceptibility profile against $S$. aureus while linezolid showed $96.55 \%$ susceptibility against gram-positive cocci (S. aureus and E. faecalis). A high degree of drug resistance was observed among gram-negative bacilli where less than $50 \%$ susceptibility was observed for cephalosporins while imipenem has shown better susceptibility profile (70\%). For empirical treatment, the Gram staining of clinical samples may provide the information about the presence and type of bacteria, thus guide the antimicrobial treatment based on institutional antimicrobial susceptibility pattern of pathogens. Nevertheless, the definitive antibiotic treatment should be culture driven and based on actual antibiotic susceptibility pattern of the cultural isolate.

The limitation of the present study was the unavailability of automated blood culture system at our institute that would have been an ideal testing tool for prolonged cultures and also would have helped in isolation of anaerobic pathogens causing PJIs so as to provide a comprehensive picture for the infecting pathogens of PJI.

\section{Conclusion}

PJI is one of the most common cause of implant failure. With an increase in the number of primary TJAs being performed each year, the total number of PJI cases will greatly increase, significantly impacting health care system and patients. Despite the developments of various techniques and diagnostic criteria, early and accurate diagnosis of PJI is still challenging. Knowledge of the local microbiological spectrum of infection and antibiogram of pathogens causing PJI's is essential for choosing appropriate perioperative antimicrobial agents and empirical antimicrobial. To reduce the culture negativity, prolonged culture incubation periods for PJI diagnosis are needed that help in increasing the sensitivity of microbiological culture thus optimizing the patient management.

\section{Funding}

This research did not receive any specific grant from funding agencies in the public, commercial, or not-for-profit sectors.

\section{Conflict of Interest}

None declared.

\section{References}

1 Sebastian S, Malhotra R, Dhawan B. Prosthetic joint infection: a major threat to successful total joint arthroplasty. Indian J Med Microbiol 2018;36(4):475-487

2 Larsen LH, Lange J, Xu Y, Schønheyder HC. Optimizing culture methods for diagnosis of prosthetic joint infections: a summary of modifications and improvements reported since 1995. J Med Microbiol 2012;61(Pt 3) :309-316

3 Koh IJ, Cho W-S, Choi NY, Parvizi J, Kim TK; Korea Knee Research Group. How accurate are orthopedic surgeons in diagnosing periprosthetic joint infection after total knee arthroplasty? A multicenter study. Knee 2015;22(3):180-185

4 Parvizi J, Tan TL, Goswami K, et al. The 2018 definition of periprosthetic hip and knee infection: an evidence-based and validated criteria. J Arthroplasty 2018;33(5):1309-1314.e2

5 Schäfer P, Fink B, Sandow D, Margull A, Berger I, Frommelt L. Prolonged bacterial culture to identify late periprosthetic joint infection: a promising strategy. Clin Infect Dis 2008;47(11):1403-1409

6 Schwotzer N, Wahl P, Fracheboud D, Gautier E, Chuard C. Optimal culture incubation time in orthopedic device-associated infections: a retrospective analysis of prolonged 14-day incubation. J Clin Microbiol 2014;52(1):61-66

7 Osmon DR, Berbari EF, Berendt AR, et al. Infectious Diseases Society of America. Diagnosis and management of prosthetic joint infection: clinical practice guidelines by the Infectious Diseases Society of America. Clin Infect Dis 2013;56(1):e1-e25 
8 Miller JM, Binnicker MJ, Campbell S, et al. A guide to utilization of the microbiology laboratory for diagnosis of infectious diseases: 2018 update by the Infectious Diseases Society of America and the American Society for Microbiology. Clin Infect Dis 2018;67(6):e1-e94

9 Collee JG, Miles RS, Watt B, Tests for the identification of bacteria. In: Collee JG, Fraser AG, Marmion BP, Simmon A, eds. Mackie and McCartney Practical Medical Microbiology. 14th ed. Edinburgh: Churchill Livingstone; 2006 131-150

10 CLSI. Performance Standards for Antimicrobial Susceptibility Testing. 27th ed. CLSI supplement M100. Wayne, PA: Clinical and Laboratory Standards Institute; 2017

11 Vaishya R, Sardana R, Butta H, Mendiratta L. Laboratory diagnosis of prosthetic joint infections: current concepts and present status. J Clin Orthop Trauma 2019;10(3):560-565

12 Tan TL, Kheir MM, Shohat N, Tan DD, Kheir M, Chen C, et al. Culture-negative periprosthetic joint infection-an update on what to expect. JBJS Open Access 2018;3(3):e0060

13 Sebastian S, Malhotra R, Sreenivas V, Kapil A, Chaudhry R, Dhawan B. A clinico-microbiological study of prosthetic joint infections in an Indian tertiary care hospital: role of universal 16S rRNA gene polymerase chain reaction and sequencing in diagnosis. Indian J Orthop 2019;53(5):646-654

14 Costales C, Butler-WuSM.Areal pain: diagnostic quandaries and septic arthritis. J Clin Microbiol 2018;56(2):e01358-e01317
15. Gunthard H, Hany A, Turina M, Wust J. Propionibacterium acnes as a cause of aggressive aortic valve endocarditis and importance of tissue grinding: case report and review. J Clin Microbiol 1994;32:3043-3045

16. Butler-Wu SM, Burns EM, Pottinger PS, Magaret AS, Rakeman JL, Matsen FA, et al. Optimization of periprosthetic culture for diagnosis of Propionibacterium acnes prosthetic joint infection. J Clin Microbiol 2011;49:2490-2495

17 Hujazi I, Oni D, Arora A, Muniz G, Khanduja V. The fate of acutely inflamed joints with a negative synovial fluid culture. Int Orthop 2012;36(7):1487-1492

18 Lalremruata R. Prosthetic joint infection: a microbiological review. J Med Soc 2015;29:120-128

19 Esposito S, Leone S. Prosthetic joint infections: microbiology, diagnosis, management and prevention. Int J Antimicrob Agents 2008;32(4):287-293

20. Salgado CD, Dash S, Cantey JR, Marculescu CE. Higher risk of failure of methicillin-resistant Staphylococcus aureus prosthetic joint infections. Clin Orthop Relat Res 2007;461:48-53

21. Peel TN, Cheng AC, Buising KL, Choong PF. Microbiological aetiology, epidemiology, and clinical profile of prosthetic joint infections: are current antibiotic prophylaxis guidelines effective? Antimicrob Agents Chemother 2012;56(5):2386-2391 